\title{
Reivindicación de la "cultura negra" y la denuncia de discriminación racial en la experiencia del Centro de Estudios Afrocolombianos y el Centro para la Investigación de la Cultura Negra (CIDCUN)*1
}

\author{
Lery Daniela Munar Espinosa ${ }^{2}$ \\ Forum des Images, Paris, Francia \\ ORCID: 0000-0001-5680-003X
}

Recibido: 23 de enero de 2020

Aceptado: 20 de marzo de 2020

\section{RESUMEN}

A partir de los años setenta, la creación de iniciativas para confrontar un panorama escaso en materia de conocimiento sobre la historia y legado de la gente negra ${ }^{3}$ en Colombia se caracterizó por la aparición de ciertos grupos y centros de estudio que expusieron la discriminación racial como un problema estructural y vigente en la sociedad colombiana. Los miembros de estos espacios se organizaron de manera voluntaria en ciudades capitales como grupos y centros de estudio. Este artículo busca señalar cuáles fueron los elementos que ubicaron al Centro de Estudios Afrocolombianos y al Centro para la Investigación de la Cultura Negra como espacios de referencia con relación a su proyecto de confrontar dicha marginación estructural liderando la reivindicación de la "cultura negra" a partir de su afirmación étnica. Así, primero presento las denuncias inaugurales sobre la vigencia de la discriminación racial desde la actividad asociativa de la gente negra en Bogotá; luego expongo cuáles fueron las motivaciones que promovieron la creación del CEA y el CIDCUN y, finalmente, los principales puntos de convergencia y disenso en la proyección de sus posturas, objetivos y propuestas durante sus primeros años de actividad.

Palabras clave: Discriminación racial, actores colectivos, “cultura negra”, reivindicación, gente negra.

\footnotetext{
*Vindication of "black culture" and the visibility of racial discrimination : the experience of Centro de Estudios Afrocolombianos and Centro para la Investigación de la Cultura Negra (CIDCUN)

${ }^{1}$ El presente artículo hace parte de una investigación más extensa a propósito de la construcción de experiencias asociativas y espacios de sociabilidad en contra de la discriminación racial en el país desde la voz de actores liderados por gente negra en Bogotá.

${ }^{2}$ Historiadora e Investigadora egresada de la Universidad del Rosario y miembro del semillero de investigación "Historias conectadas". Estudios en técnicas de traducción en la Universidad Nacional de Colombia. Asistente en el área de comunicación y archivos en el Forum des Images, Paris, Francia.

${ }^{3}$ Emplearé el término gente negra para referirme a ese énfasis en la identidad racial al que hacen referencia dichos actores colectivos para nombrar a "la raza negra" o al "negro", en el marco de su denuncia. Distinción "racial" que la gran mayoría de estos espacios ubica con los términos "negro" o de "raza negra" y en algunos "afrocolombiano". El uso de los términos y categorías para nombrar a la gente negra en los discursos de estos espacios señala a su vez, los cambios y continuidades en la forma de concebir esa "membrecía racial".

La dinámica de migración de estudiantes, profesionales e intelectuales negros (en su gran mayoría), venía haciéndose visible desde los años cuarenta, como bien lo expone el historiador Pietro Pisano al analizar las experiencias colectivas del "Club del Negro" (1943) y el Centro de Estudios Afrocolombianos (1947), en relación con la crítica que estos actores plantean frente al equilibrio de las relaciones raciales en Colombia. Ver en Pietro Pisano, 2012.
} 


\section{ABSTRACT}

By 1970s, proposals for initiatives to confront the lack of knowledge about the history and legacy of black people in Colombia were characterized by the emergence of certain groups and research centers with people who denounced racial discrimination as a structural and current problem in Colombian society. The members of these spaces were organized in a spontaneous manner in capital cities as groups and research centers. I would like to point out the elements that placed Centro de Estudios Afro-Colombianos (CEA) and Centro para la Investigación de la Cultura Negra (CIDCUN) as reference spaces according to their project to confront the structural marginalization of black people leading the vindication of the "black culture" based on its ethnic affirmation. Thus, in a first moment I present the inaugural racial discrimination denounces made by the first collective actors of black people in Bogota, later, I expose which were the motivations that promoted the creation of the CEA and the CIDCUN, and, finally, the main convergence dissent points related to the projection of their positions, objectives and proposals during their first years of activity.

Key words: Racial discrimination, collective actors, "black culture", vindication, black people.

\section{INTRODUCCIÓN}

El negro no solo es tambor, canto, música, deporte, danza y movimientos acelerados por la euforia cadencial de sus ritmos; no, en todo aquello muestra mucho de todo aquello que no le ha sido expresar de otra manera. Cobremos la confianza perdida en nosotros. Aprendamos a tener fe en nosotros mismos, clamaba con insistencias M. Garvey. No somos África, pero nos encontramos pigmento-geográficamente respaldados por una cantidad de habitantes que hacen posible pensar en el concepto de Negritud como bandera étnica inicial en el logro de nuestros objetivos; de allí la importancia de crear nacionalmente las condiciones apropiadas, no para que el negro baile, ya que sabido es que el negro baila y muy bien: lo que queremos en el mejor de los casos es no quedarnos bailando. [...] A ese nivel somos buenas personas para todo el mundo porque con ello circunscribimos lo que el blanco quiere seguir viendo en nosotros, lo pararemos en la raya y le diremos que no. Vamos por la oportunidad que nos han negado. (Smith-Córdoba, 1976: 5)

Este fragmento publicado en la revista Negritud, primer medio de comunicación que fundó en 1975 el Centro para la Investigación de la Cultura Negra (CIDCUN), expone una clara oposición y denuncia frente al estereotipo sobre el que se edificaba la representación de la gente negra según Amir Smith Córdoba, su director. La incomodidad que suponía una imagen restringida de las capacidades y actividades que el autor enunciaba como lugares comunes para "el negro" en la sociedad, se extendía para otros territorios que trascendían el referente nacional a través de una "bandera étnica inicial": la Negritud como punto de convergencia conceptual para confrontar la dominación del "blanco". Al concluir con un llamado a la población en términos de "comunidad" a partir de un vínculo étnico, se hace evidente una preocupación por la difusión de estas reflexiones para sentar la base de una voluntad de cambio frente a las condiciones de discriminación de la gente negra en el país. 
Si en 1975 esta revista, descrita en su primera edición como "una entidad jurídicamente constituida en Bogotá, con fines no lucrativos, que persigue estudiar lo que ha sido el carácter desenvolvente de la Cultura Negra en Colombia, y a nivel histórico-geográfico, el aporte del negro en la constitución de la identidad nacional" (Negritud, 1976:10), ya incluía este tipo de reflexiones, es preciso preguntarse por los motivos que convocaron a sus miembros a la creación de mecanismos y espacios específicos para analizar y visibilizar la importancia de la "cultura negra" desde una ciudad como Bogotá. Tanto el Centro de Investigación para la Cultura Negra como el Centro de Estudios Afrocolombianos (en adelante CIDCUN y CEA), fueron inaugurados en la capital del país en 1975.

A partir de los años setenta, la aparición de diversos actores colectivos fundados por gente negra contribuyó notablemente a la exposición de la discriminación racial en Colombia como una forma particular en la que la desigualdad social había afectado históricamente a la gente negra en el país. Estos actores colectivos se fundaron como centros de estudio o movimientos, liderados por personas que compartían el interés de "asociarse" de manera voluntaria para manifestar sus dudas y opiniones sobre dos temas centrales: los aportes de la gente negra en la historia nacional y la hasta entonces poco mencionada situación de discriminación racial en Colombia.

Aunque estos actores también desarrollaron actividades en diferentes puntos del país, las sedes de espacios como el CEA y el CIDCUN se ubicaron en Bogotá. Así, "alzar la voz" en colectivo desde la capital, a partir de los años setenta, resulta una particularidad en la forma en la que estos actores dieron a conocer sus posturas y reflexiones, en el marco de un contexto que, como veremos enseguida, les permitió cuestionarse por la discriminación racial con relación a diferentes dimensiones geográficas del problema. De esta manera, al acercarme a algunos mecanismos con los que el CEA y el CIDCUN erigieron sus objetivos, posturas y actividades, señalo cuáles fueron los elementos que los ubicaron como espacios de referencia en cuanto a su proyecto para confrontar la marginación histórica de la gente negra.

De esta manera, busco ahondar en los puntos de convergencia y disenso más relevantes durante los primeros años de su actividad colectiva, para así contribuir a la diversificación de una lectura de esta etapa "embrionaria" del movimiento social afrocolombiano en la que se suele destacar el lugar de estos actores desde una perspectiva teleológica y conmemorativa. Lo anterior, implica acercarse al tipo de representaciones de lo "negro" desde el discurso y las prácticas que implementaron estos actores, en un intento por esclarecer un poco más la relevancia que tuvo, en la época, definir la distinción política, intelectual y cultural de sus proyectos en el marco de dicha movilización colectiva contra el racismo. ¿Qué contenidos se discutían en estos espacios y cómo los movilizaron? ¿Qué acuerdos y desacuerdos se hicieron visibles en este proceso?

A continuación, oriento esta discusión en tres momentos: en primer lugar, señalo las denuncias inaugurales sobre la vigencia del racismo desde la actividad asociativa de la gente negra en Bogotá. En segundo lugar, expongo cuáles fueron las motivaciones que promovieron la creación del CEA y el 
Lery Daniela Munar Espinosa

CIDCUN y, posteriormente, los principales puntos de convergencia y disenso en la proyección de sus posturas, objetivos y propuestas durante sus primeros años de actividad.

\section{I.¿Por qué pensar la actividad asociativa de la gente negra desde Bogotá?: primeras denun- cias en contra de la discriminación racial desde la actividad colectiva}

Bogotá es una ciudad relevante en la historia del movimiento social afrocolombiano. Desde los años cuarenta, quienes hoy en día son considerados como la "generación de los pioneros", fueron los primeros en manifestar una postura abierta en contra de la discriminación racial como un actor colectivo desde la capital del país. Dos eventos en particular evocan este referente inaugural de una suerte de "despertar de conciencia" para las reconstrucciones históricas del movimiento: el Día del Negro (1943) y la creación del Centro de Estudios Afrocolombianos (1947). El periódico El Tiempo registró lo que ocurriría en el "Día del negro", fecha en la que se inauguró el Club y se expusieron las bases de su orientación, un día antes de los eventos:

[...] Numerosos estudiantes e intelectuales de color, residentes en nuestra ciudad, se congregarán en una reunión íntima que tendrá un admirable significado y que será una verdadera velada de arte negro. La han organizado don Natanael Díaz, estudiante negro del norte del Cauca, y varios compañeros suyos de las facultades de derecho, de medicina y de los colegios de segunda enseñanza. Sobre esta fiesta nos dijeron anoche:

-Consagraremos algunas horas a pensar- en círculo fraternal- en todos los hombres negros de Colombia y el mundo. Celebraremos nuestra fiesta sencilla y cordialmente. Recitará uno de nosotros varios poemas de Candelario Obeso y de Jorge Artel: leeremos un capítulo de la novela "Sangre negra" de Richard Wright; escucharemos varios discos de Marian Anderson; guardaremos un minuto de silencio en honor de Washington Carve, el más grande sabio norteamericano, y luego... luego bailaremos cumbia, tocaremos maracas y gozaremos de nuestra alegría negra.

Un club. En el curso de algunos días se constituirá en Bogotá un club de hombres de color. El señor Díaz y sus compañeros se proponen formar una biblioteca, organizar varias conferencias y actos diversos y constituir en nuestra ciudad un verdadero hogar de los negros colombianos. (El Tiempo, 1943: 1).

Así, el 21 de junio de 1943, un grupo de estudiantes y profesionales en su mayoría provenientes de Quibdó, Buenaventura, Puerto Tejada, Cartagena, entre otras ciudades del Pacífico y el Caribe, protagonizaron una marcha en el centro de Bogotá que tenía por objetivo manifestar la inconformidad en contra de la situación que vivían "sus hermanos de la raza negra" en los Estados Unidos (El Tiempo, 27 de junio de 1943: 15). Este mismo día fundaron el "Club del Negro" y proclamaron el "Día del Negro". Entre los miembros que lideraron y dirigieron estos eventos se destacan los hermanos Delia y Manuel Zapata Olivella, Natanael Díaz, Marino A. Viveros, Helcías Martán Góngora, Víctor M. Viveros, Arquímedes Viveros, entre otros. 
Si bien el tono celebratorio y pacífico que acompaña este registro del "Día del negro" no sugiere exactamente un levantamiento en contra de la discriminación racial, el detalle de los elementos mencionados (música, literatura, poesía y baile) y el énfasis en sucesos y personajes del pasado, así como la proyección en el futuro de un "espacio para los negros colombianos", da pistas para responder a la pregunta por la forma en que este sector de la población percibió la necesidad de destacar "el aporte de la raza negra" ante un panorama de discriminación racial en los Estados Unidos.

Así, aunque la visibilización de una postura en contra de la discriminación racial hacia la gente negra puede rastrearse desde las primeras décadas del siglo XX en espacios como la prensa ${ }^{4}$, en función de las regulaciones migratorias que impartió el gobierno para promover la llegada de migrantes de ciertas nacionalidades y obstaculizar el ingreso de otras ${ }^{5}$, fue en el marco de esta manifestación pública por las calles de la capital que, por primera vez, un grupo de personas que se identificó como "miembros de una raza" (El Tiempo, 20 de junio de 1943: 5), visibilizó su interés por exaltar creaciones artísticas hechas por personajes "negros" y, a su vez, la inconformidad en contra de la discriminación racial hacia la población. ${ }^{6}$

En estos términos, revisar los motivos para "reunirse" en Bogotá, bajo la forma de un actor colectivo a partir de su vínculo "racial", implica destacar la migración hacia la capital como una característica que este sector de la población compartió con quienes decidieron crear grupos y centros de estudio enarbolando las banderas de la "cultura negra" treinta años después. Los miembros del Club del Negro eran estudiantes o profesionales que se habían desplazado a la capital del país en busca de mejores oportunidades laborales y profesionales ${ }^{7}$. Según la detallada investigación del historiador Pietro Pisano a propósito de los liderazgos políticos "negros" de mediados de siglo en el país, la relación que estableció este sector de la población en Bogotá, además de este evidente interés por crear espacios de visibilización de los aportes de personajes "negros" destacados en el arte, la literatura y la música, y construir escenarios de intercambio intelectual al respecto, también se basó en el interés por denunciar las condiciones de desigualdad y abandono del Estado que, líderes como Natanael Díaz o Diego Luis Córdoba, identificaron en relación con sus departamentos de origen (Chocó, Valle del Cauca, Bolívar, entre otros). Así, otro escenario para pensar la visibilización de la percepción de la desigualdad en relación con las tensiones raciales en la época, fueron las denuncias de dichas inequidades en el Congreso de la República. ${ }^{8}$

\footnotetext{
${ }^{4}$ Un trabajo reciente del profesor Oscar Perdomo Gamboa, muestra la representación gráfica de la gente negra desde las caricaturas desde el siglo XIX al XXI. Oscar Perdomo Gamboa, 2017.

${ }^{5}$ Ver "La Inmigración Negra No Será Aceptada”, Diario de la Costa, Cartagena, 25 de mayo de 1923: 1; “África para los Africanos”, ABC, Quibdó, 1 de mayo de 1926: .2; "Una evocación de Langston Hughes", El Mercurio, 29 de junio de 1932: 6.

${ }^{6} \mathrm{La}$ investigación de historiadores como Francisco Flórez y Pietro Pisano, es fundamental para dar cuenta de estas tensiones en las relaciones raciales durante las primeras décadas del siglo XX. Ver Francisco Javier Flórez Bolívar, 2015 y Pietro Pisano, 2012.

${ }^{7}$ La dinámica de migración de estudiantes, profesionales e intelectuales negros (en su gran mayoría), venía haciéndose visible desde los años cuarenta, como bien lo expone el historiador Pietro Pisano (2012) al analizar las experiencias colectivas del "Club del Negro" (1943) y el Centro de Estudios Afrocolombianos (1947), en relación con la crítica que estos actores plantean frente al equilibrio de las relaciones raciales en Colombia. ${ }^{8}$ En palabras de Pisano (2012), "la cuestión racial fue un elemento clave para pensar la adscripción política de la gente negra", con relación a las reclamaciones y propuestas que expusieron algunos representantes del Chocó y el norte del Cauca desde su filiación al partido liberal. En términos generales, estas incluían el tema de la subordinación de la población negra en términos de clase "ricos (blancos) y negros (pobres)" pero no se hizo hincapié en una señalarla como un problema transversal a la misma.
} 
Lery Daniela Munar Espinosa

En estos términos, la "generación de pioneros" puso sobre la mesa la discusión sobre la visibilidad de la gente negra con relación a su papel en la construcción de una nación democrática. Siete días después de la declaración del "Día del Negro", la junta directiva del Club publicó en el periódico El Tiempo su manifiesto. Desde el principio hasta el final, se habló del "pueblo negro de América" y del sufrimiento del "negro" como un punto en común de la población a nivel continental. Respecto a la situación de discriminación que estaban atravesando los afroamericanos se hizo referencia a la democracia colombiana como un sistema de gobierno que, a diferencia de sus vecinos del norte, sí les permitía habitar una ciudadanía "más igualitaria" señalando que Colombia era la "patria de José Hilario López" (El Tiempo, 27 de junio de 1943: 15). Lo anterior, ubicaba a la esclavización como un punto en común para justificar su denuncia: la deuda que tenían los gobiernos en América en reconocer los aportes de "la raza negra" a la nación. En sus palabras, "[...] no hay lugar de América en donde el negro no estructurara su doliente sacrificio al igual que su canción, en orden al florecimiento de la humanidad”. (El Tiempo, 1943: 15)

Así, aunque en la postura del Club se reconocían ciertas convergencias históricas para la población negra del continente, esto no se articuló con una denuncia que situara a Colombia como un país en el que la discriminación racial también era un problema que continuaba perpetuando la desigualdad hacia la gente negra. En este sentido, ese interés inicial por "reunirse" para crear espacios de discusión y conmemoración a partir de dicha "membrecía racial", poco a poco fue edificando un diálogo "a varias escalas" en razón de la necesidad de visibilizar los aportes de la "raza negra" como respuesta a una marginación histórica que resultaba transversal para varios países del continente.

Es allí donde el interés por construir conocimiento a partir de la historia de la población y su legado cultural en el país y el continente permite identificar los matices que van tomando esas primeras denuncias expresadas en términos de "solidaridad racial". En otras palabras, la ausencia de conocimiento sobre la historia de la "presencia negra" en el país, poco a poco, se identificó como un obstáculo para definir el aporte de esta "raza" a la construcción de la nación.

Cuatro años después de esto eventos de 1943, bajo el liderazgo de Manuel Zapata Olivella y en compañía de la mayoría de las personas que crearon el Club, se fundó el Centro de Estudios-Afrocolombianos. Con el objetivo inicial de "indagar sobre el aporte de la raza negra a la cultura nacional y señalar cómo le ha retribuido América", la revista Semana destacó su inauguración a partir de la exaltación de un perfil intelectual de Manuel Zapata Olivella:

Ha iniciado tareas en Bogotá, bajo el consejo del Instituto Etnológico, un Centro de estudios afro-colombianos. Se propone, como otros similares ya existentes en Cuba, Brasil, Méjico y los Estados Unidos, indagar sobre el aporte de la raza negra a la cultura nacional y señalar cómo le ha retribuido América: Cuando el pequeño pero entusiasta grupo se reúne a leer documentados trabajos sobre aspectos antropológicos, etnográficos, históricos o lingüísticos del negro colombiano, es un alto, simpático y persuasivo miembro del Centro y de la raza interesada, quien preside la discusión. (Semana, 1947: 32) 
En este breve fragmento se esclarece que el objetivo principal del CEA era la construcción e intercambio de conocimiento sobre la gente negra a partir de la investigación histórica y antropológica. Aunque no es posible indagar sobre las investigaciones propuestas debido a la inexistencia de fuentes, es preciso destacar cómo el liderazgo de Zapata Olivella resultó un elemento central para ubicar la inauguración del CEA como un actor pionero en el panorama intelectual de la época. Al finalizar este perfil titulado "Deudas con el África", en el que se expone el curso de sus viajes para llegar al barrio de Harlem en la ciudad de Nueva York, se le describe como un "novelista inédito" que buscaba "fijar el papel de la raza negra" en Colombia:

El mismo que hoy estudia en la Facultad de Medicina ( $6^{\circ}$ año) y que busca fijar el papel de su raza en Colombia, es responsable de dos originales a máquina que duermen en su modesta habitación, bajo el polvo, porque no llega el editor. "Tierra Mojada", una novela sobre la intervención de un político para expulsar de sus tierras a cultivadores de arroz en el bajo Sinú. "Benkos Bhíos" [sic], un argumento cinematográfico, sobre un negro colombiano que se reveló contra España, en el siglo XVI. Y naturalmente, el señor Zapata podría hacer, en mil hojas, una novela de aventuras: su vida. (Semana, 1947: 32)

De esta manera, el énfasis en la trayectoria de Zapata Olivella en el marco de la inauguración del CEA planteaba el reconocimiento de lo "intelectual", de su liderazgo y de la orientación de este innovador espacio, como la perspectiva desde la que se estaba abordando la visibilización del legado de la gente negra en el país. Es por esto que, aunque el CEA no prosperó como se esperó en ese entonces, su adscripción a una institución pionera en el desarrollo del conocimiento antropológico en Colombia como el Instituto Etnológico Nacional ${ }^{9}$, explica por qué ese interés en explorar las características de la población implicó la descentralización del trabajo colectivo desde la capital, y con ello, podemos identificar por qué existe una suerte de "salto cronológico" entre los años cuarenta y setenta, al rastrear la visibilización de la "cultura negra" y la denuncia de la discriminación racial desde Bogotá en las reconstrucciones del movimiento social afrocolombiano.

\section{Los años setenta: el contexto propicio para la aparición de espacios liderados por gente negra en contra de la discriminación racial}

Es preciso destacar que uno de los puntos más relevantes de la investigación que recoge este aporte, fue identificar que los estudios antropológicos e históricos, así como la creación literaria, artística y musical, fueron los escenarios desde los cuales diferentes líderes, investigadores y autores visibilizaron a la población negra en el país durante dicho "salto cronológico". Si bien durante este periodo no se organizaron como actores colectivos, es necesario recorrer las trayectorias de personajes como Rogerio Velásquez, Aquiles Escalante, Delia y Manuel Zapata Olivella, Arnoldo Palacios, Jorge

\footnotetext{
${ }^{9}$ Fundado en 1941, (tres años después de que entrara en funcionamiento el Servicio Arqueológico Nacional), el Instituto fue el ente desde el cual se promovieron las investigaciones en antropología dirigidas en su gran mayoría, por una generación de investigadores extranjeros y locales como Paul Rivet, Reichel Dolmatoff, Guillermo Hernández de Alba, entre otros, quienes estaban mayormente interesados en la exploración arqueológica e histórica de los pueblos pre-hispánicos e indígenas del país.
} 
Artel, entre otros, para no desvirtuar un proceso de casi tres décadas en el que sus aportes situaron el interés por generar conocimiento sobre la historia de la presencia negra en el país como indicio de su denuncia: la marginación de la población en escenarios académicos, culturales y políticos. ${ }^{10}$ De ahí que sus contribuciones hayan sido referentes para los fundadores del CEA y el CIDCUN, quienes, a partir de los años setenta, situaron el estudio de las causas y consecuencias de la discriminación racial hacia la gente negra como una prioridad en sus agendas de trabajo. Un ejemplo de ello fue la mención de dos antropólogos destacados en el marco de la reinauguración del CEA en 1975 por parte de Manuel Zapata Olivella:

Investigadores como Rogerio Velásquez y Aníbal Escalante, precursores de los estudios socio-antropológicos respectivamente en el litoral Pacífico y Atlántico, lograron adelantar sus importantísimas investigaciones con sacrificios de toda índole. Todas estas razones le restaron impulso al Centro de Estudios Afro-Colombianos, quedando reducida su existencia a un permanente anhelo, que nunca decayó entre sus fundadores. (Boletín Informativo $\mathrm{N}^{\circ}$ 1, 1975: 3)

En el boletín $N^{\circ} 1$ del Centro de Estudios Afrocolombianos, Zapata Olivella expuso que las actividades de "los pioneros" a propósito de la visibilización de la identidad racial de la gente negra, para los años cuarenta, se vieron truncadas por un contexto social en el que estas reflexiones "de avanzada" no tuvieron la resonancia suficiente. Casi 30 años después, hizo referencia a un "presente" que sí posibilitaba la investigación y difusión de estudios sobre la presencia negra en Colombia como respuesta a las denuncias del racismo a escala global:

Con objetividad puede hablarse de una "segunda época" ya que en los inicios del Centro -1943- ocurrieron en circunstancias que pudiéramos llamar de procesos dentro del medio investigativo en Colombia. [...] Sin embargo, puede afirmarse que los gestores de la creación del Centro de Estudios Afro-Colombianos se adelantaban sustancialmente a las ideas e inquietudes sobre los aportes del negro a nuestra nacionalidad pues en esos tiempos, resaltar la contribución del negro o del indio dentro del concepto de la colombianidad, era interpretado como erupciones racistas en una sociedad en la que el silencio sobre la etnia era una tácita identificación con los valores hispánicos. Este hecho y, no otro, bloqueó el entusiasmo de los jóvenes fundadores, quienes fueron acusados de levantar una causa que intentaba introducir fieras luchas raciales en Colombia.

Desde luego que las conciencias más abiertas de la época, especialmente de sociólogos, antropólogos y demás estudiosos de las ciencias sociales y políticas, acogieron y dieron su decidido apoyo a la naciente organización. Pero a las críticas abiertas o solapadas, se sumó la carencia de recursos económicos para adelantar las investigaciones en zonas rurales donde se había concentrado la mayor parte de la población colombiana. Investigadores como Rogerio Velásquez y Aquiles Escalante, precursores de los estudios socio-antropológicos respectivamente en el litoral Pacífico y Atlántico, lograron adelantar sus importantísimas investigaciones

${ }^{10}$ Ver en "Los aportes del negro": punto de encuentro de ciertas trayectorias intelectuales a mediados de siglo" Lery Daniela Munar Espinosa (2019). 
con sacrificios de toda índole. Todas estas razones le restaron impulso al Centro de Estudios Afro-Colombianos, quedando reducida su existencia a un permanente anhelo, que nunca decayó entre sus fundadores.

El desarrollo histórico, sin embargo, trabajó favorablemente a la iniciativa. El proceso de descolonización africana; el creciente desarrollo de la antropología cultural; las luchas reivindicadoras del pueblo negro norteamericano; el auge de estudiantes negros en nuestras universidades y las investigaciones realizadas por los africanistas colombianos y extranjeros en nuestro medio han creado bases sólidas para reiniciar los pasos dados por el Centro en una atmósfera mucho más propicia para los objetivos trazados hace treinta años por sus primitivos fundadores. Puede hablarse, pues, más que de una "segunda época", de viejas aspiraciones (Boletín Informativo $\mathrm{N}^{\circ}$ 1, 1975: 3).

Lo primero que llama la atención es que Zapata ubicó la motivación de esta reinauguración, a manera de continuidad, desde 1943 con la ocasión de la creación del "Club del Negro" en Bogotá. Al señalar este evento como el antecedente, y explicar las causas de las interrupciones y ausencias de las actividades del Centro, Zapata Olivella daba a entender que la motivación para reunirse había sido más fuerte que los obstáculos que por treinta años impidieron el desarrollo de los objetivos propuestos. Es decir, esa "membrecía racial" entendida desde la actividad de estos actores de los años cuarenta, fue un llamado para que la gente negra identificara la importancia de sus aportes en la construcción de la democracia en Colombia. De ahí que estos eventos, tanto en la reinauguración del CEA como en su testimonio personal, sean recordados con un tono de nostalgia y conmemoración:

[...] - ¡Vivan los negros! ¡Abajo la discriminación racial! ¡Protestamos por los linchamientos de nuestros hermanos de raza en los Estados Unidos! Silenciosos, sorprendidos, veían marchar el puñado de estudiantes con los libros bajo el brazo, sombreros de fieltro, paraguas y corbatines rojos. Sí, indudablemente éramos negros, los puños levantados desafiantes:

- ¡Viva el áfrica del año dos mil!

[...] En el centro de la ciudad invadimos los cafés donde intelectuales, comerciantes, ganaderos, universitarios y desocupados se congregaban a saborear el tinto. Natanel Díaz, fogoso orador, se subía a una mesa y arengaba. El coro altisonante de sus compañeros aplaudíamos y cantábamos: ¡Ay mamá Inés! ¡Ay mamá Inés! ¡Todos los negros tomamos café!

[...] Tras escuchar algunos aplausos salíamos a la calle cada vez más firmes, más lúcidos, más negros. La protesta que no dejó mayores huellas en las mentes alienadas de negros, indios y mestizos, si logró profundas cicatrices en quienes la iniciamos. (Zapata Olivella, 1990: 186) 
Lery Daniela Munar Espinosa

Además de evocar estos eventos, Zapata Olivella mencionó la "pertinencia" del contexto global para darle la altura y profundidad que merecía la labor que se habían propuesto décadas atrás. El interés en extender la convocatoria a investigadores que se interesaran por la exploración profunda de la presencia de la población "negra" en América, permitiría avanzar en el estudio, la sistematización y difusión de dichas investigaciones sobre "el negro" en Colombia para así incluir su visibilización en un contexto continental. De esta manera, se consolidaría el objetivo de crear materiales de calidad y referencia que permitieran dar a conocer la participación de la gente negra en la historia de la nación, y con ello, a una vía para superar esa invisibilidad histórica como un paso clave para confrontar la discriminación racial en el país. ${ }^{11}$

En el marco de dicha reinauguración, el CEA expuso un plan de trabajo orientado en tres ejes: I. Estudios Históricos. Origen y aparición del negro en Colombia; II. Estudios etnológicos del negro en Colombia, III. Influencias culturales de los grupos raciales en Colombia (Boletín Informativo $\mathrm{N}^{\circ} 1,1975$ : 10). Además de plantearse como objetivo central la caracterización etnológica del "negro" en el país, el CEA incluía una pretensión clara de articulación entre los recursos de la investigación antropológica e histórica con un plan de acción para su difusión:

La elaboración de un fichero bibliográfico de todas las publicaciones aparecidas en Colombia sobre el negro, así como también de aquellas obras y escritos aparecidos en el exterior. El Centro de Estudios Afro-Colombianos publicará periódicamente una revista en donde se presentarán los trabajos realizados por los afiliados y reproducirá en la medida de sus posibilidades todos aquellos documentos históricos que se encuentran en los archivos de la Conquista y la Colonia. Igualmente, se reproducirán los trabajos recientes que sobre el negro aparezcan en las revistas de otras instituciones similares. (Boletín Informativo $\mathrm{N}^{\circ} 1,1975: 4$ )

A partir de estos intereses, se hace evidente que la inauguración de este espacio tenía un proyecto a largo plazo: la denuncia frontal de la discriminación racial en el país -y el continente- como justificación para promover una investigación diversa y a profundidad sobre la presencia de la gente negra y su aporte a la construcción de la nación, pero ahora, desde la actividad colectiva liderada por estos actores. De ahí que los mecanismos empleados para difundir sus primeros objetivos como actores colectivos, hayan sido la organización de plataformas como congresos, seminarios y la creación de medios de comunicación, entre otros, para así exponer la pertinencia de estos estudios en un largo camino de transformación sobre la representación del "lugar del negro" en la sociedad colombiana.

En el mismo año de la reinauguración del CEA, Amir Smith Córdoba, líder y fundador del CIDCUN, expuso en el primer ejemplar de la revista Negritud -órgano difusor del centro- que este

\footnotetext{
${ }^{11}$ Nina S. de Friedemann empleó el concepto de invisibilidad para señalar el rechazo a la negación de la negridad en una sociedad gobernada por la ideología dominante de una identidad nacional mestiza; una ideología que, mientras le daba cabida a lo indígena como forma institucionalizada de la diferencia (o de la otredad) ignoraba o menospreciaba lo negro.
} 
centro era "una entidad jurídicamente constituida en Bogotá, con fines no lucrativos que persigue estudiar lo que ha sido el carácter desenvolvente de la Cultura Negra en Colombia, y a nivel histórico-geográfico el aporte del negro en la constitución de la identidad nacional" (Negritud, 1976: 10). En estos términos, se sentó claridad sobre la producción de conocimiento en función de un objetivo que implicaba dotar de nuevos elementos la percepción de la identidad nacional con relación al rol de la gente negra en la historia del país.

Según Smith Córdoba, para llevar a cabo esa empresa, era necesario estar provisto de herramientas intelectuales para entender por qué "el negro" no había podido apropiarse de su legado cultural e histórico en Colombia. Que en la primera edición del periódico Presencia Negra Smith Córdoba expusiera, que "organizarse nace de la necesidad urgente que hay de evitar que el negro siga siendo instrumento incondicional de sus manejadores" (1979: 3), sugiere que la prioridad de ampliar y diversificar el estudio de la "cultura negra", cobraba sentido en el marco de la actividad colectiva como una respuesta a la marginación estructural a la que se había sometido a la población, respecto a la comprensión de la construcción de su identidad: a partir de ese vínculo "racial", desde una perspectiva histórica.

Sin embargo, y al igual que el CEA, la promoción de este objetivo no restringía la "convocatoria" a todos aquellos que quisieran contribuir a la transformación en la representación de la gente negra del país a partir de la investigación como punto de partida:

No es una dicotomía lo que propugnamos, propugnamos un movimiento no solo de todos los negros que pueblan el país, sino de todos, sin distinción de credo, raza, origen, sexo, etc.; es decir de todos aquellos que estén predispuestos a investigar a un ancestro que ha sido sistemáticamente tergiversado [...] (Smith-Córdoba, Presencia Negra $\mathrm{N}^{\circ} 1,1979$ : 3).

En estos términos, el CIDCUN promovió un proyecto de base intelectual para estimular la apropiación de elementos que condujeran a forjar esa "conciencia étnica" como un proceso de aprendizaje conjunto. Bajo la consigna de "aprender a ser negro", el CIDCUN, además de emplear los espacios ya señalados característicos del entorno académico, se caracterizó por ser un actor que incorporó mecanismos de difusión propios que sirvieron como punto de encuentro y fuente de divulgación sobre la reflexión de la "cultura negra” y la discriminación racial: la revista Negritud y el periódico Presencia Negra.

Al señalar algunas continuidades de la motivación de carácter "intelectual" para explicar el surgimiento de estos actores colectivos, es decir, este objetivo en común por construir investigación como punto de partida para la visibilizar a la población y la discriminación racial que históricamente ha padecido, queda más claro por qué identificar la discriminación como obstáculo para el goce pleno de la ciudadanía estaba ligado a una voluntad de transformar una condición histórica de marginación e inferioridad. Por esta razón, adquirir el conocimiento sobre un pasado hasta 
entonces "desconocido", implicaba, además una fase central para apropiarse de esta "lucha", hacer de la invisibilidad epistemológica y estructural de la población una bandera que se enarbolara específicamente desde estos espacios liderados por gente negra. Esto explica por qué a partir de la revisión de los mecanismos de difusión empleados para visibilizarla, se pueden establecer hipótesis del compromiso que cada uno de estos actores tenía con el descubrimiento de la "cultura negra" como un camino a seguir para forjar una conciencia política de la población

Ahora bien, ¿en qué medida dicha prioridad por la investigación se articuló con otros elementos que motivaron la movilización cívica de la época? Un importante elemento de cohesión para los miembros de estos primeros espacios fue la discriminación racial que experimentaron en el curso de esa migración interna hacia ciudades como Bogotá. La discriminación expresada en manifestaciones cotidianas, que, según sus testimonios, no habían vivido en sus ciudades natales y de procedencia, los motivó a reunirse y crear encuentros a partir de la pregunta inicial sobre las causas de esa marginación social e inferioridad que identificaron en común.

Un segundo elemento, sin duda, obedece al contexto de movilización cívica desde la afirmación étnica a escala internacional. Los investigadores Mauricio Pardo (2001), Luis Carlos Castillo (2016) y Carlos Agudelo (2004), señalan que, la creación de estos espacios en el país no puede desvincularse de la apropiación local de las luchas por los derechos de la población en países como Estados Unidos y Sudáfrica. De ahí la constante referencia a este como "el momento indicado" para el surgimiento del movimiento social afrocolombiano. Fue en este punto en que "la creación de grupos de discusión de estudiantes intelectuales" o "las expresiones organizativas de la época", respondía la necesidad de indagar y debatir sobre la experiencia de la discriminación vivida y el interés sobre los eventos de reivindicación de los derechos de la gente negra de otros países desde una evidente curiosidad histórica.

Mire, uno pasaba por la séptima la gente se persignaba, se rascaban las rodillas, era como la presión de la discriminación dura. Una presión visual, gesticular. Bueno, eso me fue llevando a estudiar más. Me acuerdo de que en la universidad decían "vaya mata un negro y haga patria", "las negras son putas por naturaleza, las rolas por religión."12

Ahora bien, si otra particularidad en común de estos espacios fue su carácter informal y extracurricular con respecto a las actividades profesionales de sus miembros, es preciso exaltar el lugar de sus inquietudes políticas en un contexto en el que la lucha de clases dominaba la movilización ideológica de la época. La aparición de estos actores a partir de una afirmación étnica también respondía a un último elemento a integrar en la respuesta por su creación, continuidad e institucionalización: la escasa cabida que la discriminación racial tuvo como un problema a abordar desde la actividad de los movimientos políticos de izquierda de la época:

${ }^{12}$ Rudecindo Castro (comunicación personal, 28 de agosto de 2017). 
De todas formas, estaban pasando cosas en el sur (refiriéndose al Apartheid de Sudáfrica) y en el norte (al despliegue de los movimientos de derechos civiles en Estados Unidos), y pues nosotros ahí en el centro, mirábamos para ambas direcciones. Es como un caldo de cultivo que hizo que reflexionáramos sobre nuestras condiciones acá. Las preguntas por el reconocimiento al otro, en este país diverso, empezaron a calar en algunos líderes. En este proceso, yo personalmente siento que Juan de Dios Mosquera fue un actor clave. Yo siento mucho aprecio por él y lo qué el hizo desde el grupo Soweto y luego con Cimarrón, va a ser clave para el movimiento que va desarrollando después. ${ }^{13}$

Estas palabras del historiador quibdoseño Sergio Mosquera dan una imagen sobre esa "lucha a varias escalas" en la forma de concebir la reivindicación de la "cultura negra". Para esta época, las luchas anticoloniales en África, el movimiento por los derechos civiles en Estados Unidos y el Apartheid de Sudáfrica, ya eran procesos que la prensa registraba inclusive en países tan distantes a estos eventos como Colombia. Juan de Dios Mosquera, líder y fundador del Movimiento Nacional Cimarrón, señaló que en los periódicos de izquierda solían encontrarse noticias sobre el movimiento por los derechos civiles de Estados Unidos y del Apartheid en Sudáfrica, y que, aunque escasos, circulaban ciertos materiales traducidos al español sobre la actividad de líderes como Martin Luther King y Nelson Mandela. ${ }^{14}$ Así, el interés por informarse sobre la situación de las luchas cívicas en otros contextos impulsó a estos actores a abordar el análisis de la "cuestión racial" a partir del contraste de experiencias de dicha subordinación y discriminación "compartida":

Había un compañero que tenía algunos libros sobre Mandela y Sudáfrica -Eusebio - y yo, me fui interesando mucho por Martin Luther King y el movimiento de los derechos civiles en Estados Unidos. De ahí tomé una gran influencia del pensamiento y el método de Malcom X y las organizaciones del poder negro, los estudiantes, me influenciaron mucho. Yo mentalmente me trasladaba a los Estados Unidos y trataba de entender para aplicar en la realidad colombiana. Para ese tiempo, mi facultad era una facultad comunista. Los profesores estudiaban en Moscú y eran miembros del partido comunista, militantes, y en la universidad el aprendizaje en torno a toda la teoría marxista, la lucha de clases, también me permitieron entender dónde estaban los conceptos étnicos, la población afrocolombiana, dónde estaba el tema racial, la discriminación tanto en la izquierda como en la derecha del país. O sea, que no estaba. Eso reafirmó más mi convicción de que teníamos que construir pensamiento propio. ${ }^{15}$

En este sentido, Mosquera planteó que la teoría marxista y la tendencia ideológica de izquierda contribuyeron a ese "primer despertar" con relación a la comprensión del problema racial desde el marco de estudio de la lucha de clases. Así, se seguía afirmando la motivación intelectual como punto de encuentro para el desarrollo de una reflexión estructural sobre la discriminación en el

\footnotetext{
${ }^{13}$ Sergio Mosquera Mosquera (comunicación personal, 25 de agosto del 2017).

${ }^{14}$ Ver "Panorama del África liberada". Voz Proletaria No. 932 Del 2 al 8 de junio de 1977, p, 2; "Auge del racismo en EE.UU" Voz Proletaria No. 937 del 7 al 13 de julio de 1977, p, 12 "El apartheid como un sistema de gobierno", "Más violencia racista” Voz Proletaria No. 944 del 25 al 31 de agosto de 1977, p, 12.

${ }^{15}$ Juan de Dios Mosquera Mosquera (comunicación personal, 27 de abril de 2017).
} 
Lery Daniela Munar Espinosa

país. Al respecto, hay que aclarar que Soweto, círculo de estudios bajo su liderazgo, nació en 1976 por parte de un grupo de estudiantes de la Universidad Tecnológica de Pereira. No obstante, al convertirse con los años en el único de estos espacios que perduró -hasta la actualidad-, con el nombre de Movimiento Nacional por los derechos civiles Cimarrón, resulta un referente transversal a la construcción de las posturas frente a la discriminación racial desde los actores colectivos en la capital, porque, además, la sede principal del movimiento decidió trasladarse a Bogotá para 1984. Ya que su poder de convocatoria en torno a la discusión inexistente sobre el problema étnico en el país se expresó desde una perspectiva teórica ligada al materialismo histórico, es preciso identificar que ese proceso de comprensión de la discriminación también constituye un punto de encuentro con actores como el Centro de Estudios Afrocolombianos y el CIDCUN.

Así, queda claro que las motivaciones que impulsaron a estos actores a organizarse de manera colectiva no implicaron un rechazo a los otros escenarios en los que también se visibilizó la presencia de la población en el país. Es más, considero que durante el periodo en el que estos espacios existieron, forjaron un puente con los espacios académicos desde la antropología, la historia, la literatura y el arte para pensar la presencia negra en el país. Esta relación de doble vía caracteriza la historia del movimiento social y de los estudios afrocolombianos.

\section{Los principales puntos de convergencia y disenso entre el CIDCUN y el CEA durante sus primeros años de actividad}

Como he expuesto, la invisibilidad antes de ser acuñada por los antropólogos durante los años ochenta, fue punto de partida para estos actores colectivos que situaron la discusión de la "cultura negra" como prioridad para transformar la representación de la gente negra en el marco de los referentes para pensar la identidad nacional. Sin embargo, es claro que dicho interés estaba ligado a la voluntad de cambio en las condiciones de abandono y desigualdad que continuaba padeciendo la población. De esta manera, el proyecto de "aprender a ser negro" promovía una representación de la población "en construcción"; es decir, siguiendo la idea de una progresión por etapas cuya fase final sería la apropiación de ese conocimiento integral de base histórica para reclamar el pleno goce de una ciudadanía sin discriminación racial. Es en este punto, en la proyección de un proyecto pedagógico, político y cultural, que tanto el CEA como el CIDCUN comparten la mayoría de sus posturas.

Quizás la ruta que más nos aproxime a la proyección del Centro de Estudios Afrocolombianos, sea el análisis de las memorias del Primer Congreso de la Cultura Negra de las Américas (1977). El reconocimiento de la vigencia de la discriminación racial en el marco de este evento celebrado en agosto de 1997 en la ciudad de Cali posicionó al Centro como un espacio cuya postura partió de una motivación intelectual que no se disoció de lo político y cultural, porque, solo mediante dicha articulación -en la práctica, refiriéndose a participantes, saberes, perspectivas- se podía erigir una estrategia para dar cuenta de una voluntad política de transformación a varias escalas: 
1. Incrementar los estudios etnográficos, sociales, económicos, psicológicos, políticos y artísticos, tendientes a ubicar al hombre negro dentro del contexto histórico que le corresponde a las Américas.

2. Replantear los valores y aportes africanos acordes con los sentimientos y aspiraciones de la propia población negra.

3. Unificar las acciones y objetivos de los investigadores de la cultura negra en nuestro continente.

4. Planificar las investigaciones futuras en base a las orientaciones y conclusiones obtenidas en el Primer Congreso de la Cultura Negra de las Américas.

5. Generar la formación de instituciones dedicadas a la investigación de los valores afro-americanos en todos los países del continente, así como estrechar los nexos y acciones de las ya existentes

6. Promover la creación de un fondo para el fomento y desarrollo de la cultura negra de las Américas.

Áreas de discusión

1. Etnia negra y mestizaje

2. Filosofía y afectividad

3. Creatividad social y política

4. Creatividad material

(UNESCO, Fundación Colombiana de Investigaciones Folclóricas, 1988: 4)

Al acercarse en detalle a los términos en los que se expresó la convocatoria, desarrollo y conclusión del evento, es posible identificar que sí había una propuesta política en función de la articulación entre diferentes disciplinas para promover una representación de la población basada en el discurso de una comunidad transnacional a favor de la visibilización de la "cultura negra". Esto ubicó el diálogo entre la investigación y la política como un elemento transversal para el cumplimiento de sus tareas. Así, lo expresó Manuel Zapata Olivella en razón al objetivo principal de este primer congreso:

[...] encauzar el espíritu investigador de las nuevas generaciones hacia el conocimiento de los valores aportados por los pueblos de etnia africana, como fundamento para fecundar e impulsar las reivindicaciones políticas, económicas, sociales y culturales de la población de origen negro de nuestro continente. (UNESCO, Fundación Colombiana de Investigaciones Folclóricas, 1988: 3) 
Lery Daniela Munar Espinosa

En estos términos, la exploración e investigación sobre la "cultura negra" "debía" traducirse a nuevas formas de proyectar planes de acción que trascendieran de los escenarios académicos. En este sentido, algunas conclusiones del evento permiten identificar que esta era una consigna prioritaria, a pesar de que aun deba explorarse cómo se materializó:

Debatiendo la situación racial en Colombia, se focalizó la ausencia de participación justa del negro en la economía y la política del país, así como las causas de la misma. Se aprobó una propuesta teniéndose en cuenta que además del trasfondo económico-social existe el elemento raza usado para negar al negro una participación debida en todos los niveles en la vida del país. (UNESCO, Fundación Colombiana de Investigaciones Folclóricas, 1988: 145)

En estos términos, si evaluamos la experiencia del CIDCUN también podemos identificar el reconocimiento del accionar colectivo como un marco institucional novedoso desde cual se podía operar integrando diferentes mecanismos para favorecer la investigación sobre la presencia negra en el país. En términos generales, el CIDCUN se diferenció de los otros actores de la época por la forma en que logró diversificar su proyecto pedagógico.

Si bien mecanismos como la revista Negritud y el periódico Presencia Negra sobresalieron como los órganos difusores de este centro, siendo este último el único medio de comunicación constante (1979-1984 y 1988) creado por estos actores, una característica central es que también fungieron como espacios de convergencia y visibilización de aportes periodísticos, literarios, investigativos, entre otros, que se adherían al proyecto en común de visibilizar a la población y sus luchas a escala regional, nacional y global. Así se registró en el primer número de la revista Negritud: "los artículos dan la opinión de sus autores, y no necesariamente la orientación oficial del movimiento. Los trabajos aquí publicados pueden reproducirse parcial o totalmente, siempre y cuando se haga conocer la fuente" (Negritud, 1976:1). Esta misma aclaración aparece de manera constante en la emisión de los números de Presencia Negra, y, con ello, se precisaba la intención del CIDCUN de proyectarse a la vez como un espacio para forjar un proyecto plural para promover la transformación de la conciencia política de la gente negra.

Adicionalmente, el CIDCUN inauguró espacios similares a los empleados por el CEA; la participación en congresos y seminarios, más específicamente el Seminario sobre Formación y Capacitación de personal docente en Cultura Negra, fueron eslabones en la cadena de la transformación educativa que requería "aprender a ser negro". No obstante, que su crítica frontal a la discriminación desde estos diferentes mecanismos se asociara con una postura radical, sugiere que la crítica que atacara la imagen de armonía racial, producto de la representación de una identidad mestiza, continuaba generando un rechazo generalizado:

En muchos círculos se comenta y con mucha insistencia la tarea adelantada por el "CENTRO PARA LA INVESTIGACIÓN DE LA CULTURA NEGRA EN COLOMBIA” con opiniones 
encontradas sobre el particular. No es de dudarlo eso sí que los más de nuestros críticos, son los mismos que les da pena cargar la revista NEGRITUD o el periódico PRESENCIA NEGRA por la calle y no quieren que se vayan a tildarlos siquiera de racistas. ¿Qué tal? ¿Y racista por qué? En realidad, son comportamientos que uno mismo no alcanza a explicarse. (Smith-Córdoba, Presencia Negra Na2, 1979: 4)

De esta manera, es preciso identificar que ambos actores coincidieron en una proyección a mediano y largo plazo en aras de llegar a alcanzar una madurez política en clave de identidad "negra". Para el CEA, la prioridad fue trazar rutas de investigación de la "cultura negra" que permitieran denunciar la invisibilidad como un punto de convergencia entre la gente negra del continente, y con ello, como actor colectivo en Colombia, sustentó que le correspondía dar cuenta de una representación de la gente negra "en construcción” y en conexión con centros homólogos a escala continental. Es decir, lo que en el boletín inaugural del Centro se propuso como "la cooperación patriótica de todos los ciudadanos para no desfallecer en la dura responsabilidad que hemos recogido de la indolencia de nuestros antecesores" (Boletín Informativo $N^{\circ} 1,1975$, p.14), en la práctica, aludía a una postura politizada del "valor de los aportes del negro", en tanto se aspiraba al "derecho de reclamar una cultura Afro-americana en los contextos mestizos de América" (UNESCO, Fundación Colombiana de Investigaciones Folclóricas, 1988:181). Con ello, la membrecía a estos espacios representaba la adherencia a una voluntad política de cambio:

La delegación colombiana presentará una proposición para que oficialmente se incorpore la enseñanza de la Historia de África en la escuela primaria y secundaria, a la par de que se exija por parte de los profesores un mayor análisis del significado de la presencia negra en nuestra comunidad a través del proceso histórico desde su arribo e integración en la vida económica, social y cultural. En el ámbito panamericano de los estudios del negro, propondremos que los organismos regionales (OEA) y los internacionales (UNESCO) patrocinen en el futuro a los Congresos de la Cultura Negra de las Américas al igual del ya existente para el estudio de los problemas del indio. Además, se hará énfasis en que la situación del negro es doblemente afligida. A la marginación cultural se le suma el marginamiento social, ya que a las comunidades negras se les mantiene en zonas incomunicadas, ahondando las injusticias dejadas por la esclavitud y los primeros decenios de la vida republicana. (UNESCO, Fundación Colombiana de Investigaciones Folclóricas, 1988: 21)

Así, el liderazgo del CEA en el marco del Primer Congreso de 1977 permite identificar una propuesta política que buscaba, desde la teoría y la práctica, la representación de la población basada en el discurso de una comunidad transnacional a favor de la visibilización de la "cultura negra". Esto ubicó el diálogo entre la investigación y la política como un elemento transversal para el cumplimiento de la misma. Sí, el liderazgo del CEA en el marco del Primer Congreso de 1977 permite identificar una propuesta política que buscaba, a partir de la investigación interdisciplinar sobre la "cultura negra" y su respectiva divulgación, transformar la representación de la población en función del referente de una "comunidad" que trascendía las distinciones nacionales. Al ser 
Lery Daniela Munar Espinosa

un punto de convergencia "explorar" los lazos o conexiones que podían fortalecer el discurso de la identidad "negra" a partir de un pasado en común por la población del continente, el diálogo con los referentes geográficos (local, regional, nacional, continental, entre otros) fue central para dicha transformación. Por esta razón, es posible plantear que la representación de la gente negra, desde la voz del CEA y el CIDCUN, se diversificó en función de la concepción y transformación del referente de "membrecía racial", de cara al interés "común" por resignificar el lugar de la "presencia negra" en la construcción de la nación. Una representación de la gente negra en clave de "comunidad" que, adicionalmente, trascendía esas fronteras nacionales por la convergencia de sus denuncias frente a la discriminación racial.

\section{CONCLUSIONES}

El interés por explorar la forma en la que ciertos sectores de la población se han organizado en forma colectiva para dar cuenta de su postura con relación a la discriminación racial, es un tema reciente en el marco de la exploración de un movimiento político cuyos rasgos identitarios reposan sobre las polémicas categorías de lo "racial" y lo étnico." En este contexto, la aparición de actores colectivos fundados y liderados por gente negra que expresaron su interés por "descubrir la cultura negra", como una necesidad para evaluar su forma de habitar la ciudadanía, abre un abanico de preguntas sobre la lectura que desde los estudios afrocolombianos se ha hecho de la fase "embrionaria" del movimiento social afrocolombiano.

En aras de aportar a este campo, priorizando un referente geográfico como Bogotá, pude establecer que la diversidad en los mecanismos y estrategias que construyeron estos actores desde la actividad colectiva y los liderazgos de sus miembros es proporcional a los elementos que integraron progresivamente a sus posturas para construir una nueva representación de la gente negra. La "membrecía racial" y los lazos o conexiones que se tejieron en el discurso de la identidad "negra", a partir de un pasado en común compartido de manera transversal en el continente, fueron referentes centrales en este proceso que inauguró, desde esta perspectiva de análisis de la asociatividad, el Club Negro en la capital en 1943 y la intención de consolidar el Centro de Estudios Afrocolombianos (1947).

Que la fundación de la mayoría de estos primeros actores colectivos haya sido en Bogotá, invita a indagar por la apropiación y selección de referentes a partir de los que se concebía la desigualdad en las relaciones raciales. Lo anterior se expresó en el interés por integrar experiencias que dieran cuenta de una idea de "comunidad" vinculada por la experiencia histórica de la esclavización y sus consecuencias en lo que respecta a la relación entre la reivindicación y el territorio de las naciones en el continente americano.

En este sentido, se explica por qué una de las motivaciones para organizarse como actores colectivos, las prácticas cotidianas de discriminación racial, se haya instrumentalizado como una denuncia para el discurso político -el de la necesidad de congregarse "para"- y no como un problema 
de investigación pertinente en el marco de esta ruta de análisis del problema social del racismo. Primero se debía abordar el estudio de la gente negra en términos de "escribir los capítulos" que le faltaban a la historia de su presencia en el continente. En esta misma línea, la escasa acogida que tuvo la discusión de la discriminación racial en el marco de los liderazgos políticos de izquierda en la ciudad, fortaleció la motivación por crear espacios que permitieran abordar la investigación y el debate desde la afirmación étnica, sin que esto desestimase ni la adherencia ideológica hacia las reivindicaciones de los sectores oprimidos en la lucha de clases, ni la escogencia del materialismo histórico como referente para desentrañar las raíces del racismo en la historia del país.

Entonces, los objetivos, el conocimiento y los planes de acción que se exponían en el marco de estas plataformas, tomando el ejemplo del Primer Congreso de Cultura Negra de las América (1977), servían para concretar la proyección de sus actividades en función del diálogo entre la investigación y la política. Así, la promoción de escenarios para crear propuestas de acción a partir de la articulación entre diversos aportes antropológicos, históricos, artísticos, literarios, entre otros, fortalecía el discurso de la reivindicación de la gente negra como un llamado a la unión para cuestionar y superar la vigencia de la discriminación racial con un poder de convocatoria que trascendía la distinción "racial”. De ahí que, en los registros de congresos, seminarios y encuentros, que promovieron actores como el CEA y el CIDCUN, un punto de convergencia central haya sido la representación de la población negra a partir de un referente de "comunidad" que tampoco estaba restringido a "límites" nacionales.

Tanto en el caso del Centro de Estudios Afrocolombianos como del CIDCUN, el interés por una investigación interdisciplinar, si se quiere, politizó una lectura sobre la difusión de la "cultura negra" durante el desarrollo de los planes de acción y la proyección misma de estos actores a mediano y largo plazo. En este sentido, el CIDCUN irrumpió en el panorama por el interés que portó en los mecanismos de difusión sobre el conocimiento que promovió. Así, "aprender a ser negro" fue la consigna de un proyecto político que suponía la interiorización de herramientas que contribuyeran a la desalienación del "negro" de una estructura que promovía un lugar marginal de la población en la forma de habitar la ciudadanía. Este aprendizaje buscó cultivar el interés por explorar, comprender y difundir la historia y legado de la presencia negra en el país para posicionar "la cultura negra" como base de una conciencia política de carácter étnico.

En estos términos, es posible señalar que el interés por crear espacios diferenciados a los ya existentes en la academia y los movimientos políticos, respondía al reconocimiento de la asociatividad como un mecanismo político para la visibilización y transformación de los problemas que, en un primer momento, identificaron a partir de la relación entre la representación marginal del "negro" en la historia de la formación de las naciones en el continente y los obstáculos que le impedían a la población apropiarse de la discusión sobre la discriminación racial como un problema vigente en el país. De este modo, queda claro que diversificar la investigación y nutrirla cada vez más, fue prioridad para establecer una vía de solución política que confrontara el 
Lery Daniela Munar Espinosa

racismo hacia la gente negra en el país y el continente. También, incluyendo la discusión por el tipo de espacios políticos "por conquistar" o crear para consolidar sus proyectos en escenarios de poder que continuaban siendo desiguales para la gente negra.

De esta manera, considero que es preciso crear estrategias metodológicas que permitan integrar, por una parte, la ambigüedad existente respecto del desequilibrio en la diversidad de fuentes, y por otra, el análisis de las escalas geográficas como herramientas en la creación de representaciones identitarias desde la afirmación étnica. En este orden de ideas, este acercamiento al problema, al igual que la investigación de la cual se deriva este texto, hace parte de una propuesta que busca darles voz a los actores que no han sido abordados con rigor desde la historia del movimiento social afrocolombiano, ya sea por no haber alcanzado una solidez en un periodo extenso de tiempo, o porque se caracterizan por un registro superficial en su paso por el panorama de la movilización colectiva de la gente negra durante el siglo XX.

\section{BIBLIOGRAFÍA}

Agudelo, C. (2004). No todos vienen del río: construcción de identidades negras urbanas y movilización política en Colombia. En Restrepo, E. y Rojas, A. (Eds.). Conflicto e (in)visibilidad: retos en los estudios de la gente negra en Colombia (pp. 173-195). Popayán: Editorial Universidad del Cauca.

Auge del racismo en EE. UU. (7 al 13 de julio 1977). Voz Proletaria, (937), 12.

Castillo, L.C. (2016). Organizaciones afrocolombianas. Una aproximación sociológica. Cali, Colombia: Universidad del Valle.

Centro de Estudios Afro-Colombianos. (1975). Boletín Informativo N 1. Bogotá, Colombia.

Congreso de Cultura negra. Instalan hoy en el Cabildo de Cali. (24 de agosto de1977). El País, 3.

Córdoba, A.S. (1980). Cultura Negra y Avasallamiento Cultural. Bogotá, Colombia: Centro para la Investigación de la Cultura Negra, MAP Publicaciones.

Deudas con el África. (7 de agosto de 1947). Revista Semana, III(42), 32.

El 24 instalan en Cali el Congreso de Cultura Negra. (9 de agosto de 1977). El País, 7.

El apartheid como un sistema de gobierno. Más violencia racista (25 al 31 de agosto de 1977). Voz Proletaria, (944), 12. 
Flórez Bolívar, F. (2015). Un diálogo diaspórico: el lugar del Harlem Rennaissance en el pensamiento racial e intelectual afrocolombiano (1920-1948). Historia Crítica (5), 101-124.

Friedemann, N. de. (1986). Estudios de negros en la antropología colombiana: presencia e invisibilidad. En: Friedemann, N. de y Arocha, J. (Eds.). Un siglo de investigación social (pp. 515-572). Bogotá: Etno.

Los negros colombianos. (20 de junio de 1943) El Tiempo, 3.

Los negros colombianos lanzan un manifiesto para la América. (27 de junio de 1943). El Tiempo, 15.

Moreno Salazar, Valentín. (1995) Negritudes. Cali, Colombia: Editores XYZ

Munar Espinosa, L.D. (2019). ¿Quién habla en nombre de quién?: Espacios de sociabilidad y experiencias asociativas de la gente negra en Bogotá (1943-1988). (Tesis de grado). Universidad del Rosario.

Panorama del África liberada. (2 al 8 de junio de 1977). Voz Proletaria (932), 2.

Pardo, M. (2001). Escenarios organizativos e iniciativas institucionales en torno al movimiento negro en Colombia. En: Pardo, M. y Archila, M. (Eds.). Movimientos sociales, Estado y democracia en Colombia, (pp. 321-345). Bogotá: Universidad Nacional de Colombia, Instituto Colombiano de Antropología e Historia

Perdomo Gamboa, O. (2017). 1000 Caricaturas afro en la historia de Colombia. Cali: Universidad del Valle.

Pisano, P. (2012) Liderazgo político “negro” en Colombia: 1943-1964. Bogotá. Universidad Nacional de Colombia. Facultad de Ciencias Humanas, Departamento de Historia.

Pulido Londoño, H. (2011) Construcción y representación de los sujetos afrocolombianos en el discurso antropológico, 1980-2005. (Tesis de maestría en Historia). Universidad Nacional de Colombia. Bogotá.

¿Qué es el "Centro para la investigación de la Cultura Negra en Colombia? (agosto 1976). Negritud, (1), 10.

Restrepo, E. (1997) Afrocolombianos, antropología y proyecto de modernidad. En: Restrepo, E. y Uribe, M.V. (Eds.). Antropología en la modernidad (pp. 225-244). Bogotá: Instituto Colom- 
Lery Daniela Munar Espinosa

biano de Antropología.

Restrepo, E. y Velandia, P. (2017) Estudios afrocolombianos: Balance de un campo heterogéneo. Tabula Rasa (27), 161-197.

Smith Córdoba, A. (1980) Cultura Negra y Avasallamiento Cultural. Bogotá: Centro para la Investigación de la Cultura Negra, MAP Publicaciones.

Smith Córdoba. A.S. (agosto 1976) Identidad del negro. Negritud, (2), 5.

Smith Córdoba. A. (enero-febrero1979) Editorial. Presencia Negra (1), 3.

Smith Córdoba. A. (abril 1979) Prejuicio: “Pequeño Burgués”. Presencia Negra (2), 4.

UNESCO, Fundación Colombiana de Investigaciones Folclóricas. (1988). Primer Congreso de la Cultura Negra de las Américas. Cali-Colombia. Homenaje a León Goutran Damas. Bogotá: UNESCO, Fundación Colombiana de Investigaciones Folclóricas, ECOE.

Valderrama, C. (2013). Folclore, raza y racismo en la política cultural e intelectual de Delia Zapata Olivella. El campo político-intelectual Afrocolombiano. Revista CS, (12), 259-96.

Valero, S. (2013). ¿De qué hablamos cuando hablamos de 'literatura afrocolombiana'? o los riesgos de las categorizaciones”. Revista de Estudios de literatura colombiana (32), 7-15.

Wabgou, M., Arocha Rodríguez J., Salgado Cassiani, A. Carabalí Ospina, J. (2012). Movimiento social afrocolombiano, negro, raizal y palenquero: el largo camino hacia la construcción de espacios comunes y alianzas estratégicas para la incidencia política en Colombia. Bogotá: Universidad Nacional de Colombia. Facultad de Derecho, Ciencias Políticas y Sociales. Instituto Unidad de Investigaciones Jurídico-Sociales Gerardo Molina (UNIJUS).

Zapata Olivella, M. (1990) ¡Levántate mulato! Por mi raza hablará el espíritu. Bogotá: Rei Letras Americanas. 